\title{
Practice Note: Responding to Civic Conflict: Developing Intergroup Dialogue Co-facilitators
}

\author{
KRISTIN CHUNG-MEI LENSEN
}

MARK CHESLER

NANCY BROWN

A large U.S. city (called here, Coastal City), with a liberal milieu and a history of racial and ethnic conflict, was elected several years ago to increase its ability to deal with racial conflict. The city chose the intergroup dialogue (IGD) method. Here we examine IGD and how it differs from some other conflict response approaches-including the program's purpose, design, initial implementation, and lessons learned in this enterprise. This article describes a program to train intergroup dialogue co-facilitators, engaging a civic process to improve race relations in a large urban setting.

\section{Context and History}

R acial separation, misunderstanding, bias, inequality, and conflict are R among the most stubborn features of the U.S. landscape. This is no less true of Coastal City, one of the nation's whitest (84\%) cities, which has experienced particular problems between the largely white police department and local communities of color. Following prolonged, significant conflict about police racial profiling, the municipal administration created a committee to "facilitate dialogue between the police and the community."

We appreciate the cooperation and commitment of Coastal City's elected officials and staffs, community leaders, and all IGD co-facilitators and participants. 
This committee did not achieve the reconciliation or collaborative action sought by participants of color or white participants.

Eventually, in 2009, a unit of Coastal City's government retained the lead author to design an innovative response to the city's racial conflict. Lensen has an extensive record as a trusted resource for public and private organizations in the area. She recommended intergroup dialogue as an approach, using volunteer community members as co-facilitators, and brought Chesler and Brown into the program. Lensen is an Asian American woman in her fifties, Chesler a white man in his seventies, and Brown a white woman in her sixties.

\section{What is Intergroup Dialogue?}

Intergroup dialogue (IGD) ${ }^{1}$ is a fast-emerging practice in educational, corporate, and civic efforts to deal with intergroup conflict. Its purposes are:

- To increase understanding of individuals' locations in societal systems of advantage and disadvantage

- To increase understanding among groups of people who usually and with consequence are in conflict

- To help everyone understand how societal structures of inequality affect individual, group, and intergroup behavior

- To foster alliances across group boundaries, creating opportunities for collective action-taking to reduce social injustice

IGD has much in common with several other approaches to problem solving or peace- and justice-making. It also differs from them in important ways. IGD differs from most truth-and-reconciliation processes in its emphasis on the mutual responsibility and vulnerability of all parties, and in its thrust toward action-taking. IGD differs from most mediation efforts as it prioritizes building relationships over resolving issues. IGD differs from arbitration and legislative efforts as its participants themselves are involved in all decisions without external-authority intervention. IGD differs from most community organizing as it promotes understanding and interaction among groups prior to action-taking.

While some use the terms dialogue and intergroup dialogue interchangeably, we see crucial distinctions between those dialogue models, which focus on education and prejudice reduction, and IGD's emphasis on 
building lasting relationships and alliances for system change. The most salient differences are IGD's explicit foci on the realities of privilege and oppression, on the impact of race and ethnicity and other social group identities on one's life experiences and behaviors, and its call to collaborative action. Other forms of dialogue are suitable for other purposes.

IGD requires facilitative leadership of sustained interaction within groups of twelve to sixteen. These groups involve close-to-equal numbers of participants, between those who have privileged or advantaged status in society and those who are from relatively disadvantaged or oppressed groups. The equal-numbers strategy makes it more likely that, when customary patterns of domination and subordination manifest in the IGD, members can recognize their impact and create new, more egalitarian behaviors. The twelve-to-sixteen participant count makes the group large enough to have meaningful intragroup and intergroup differences present, yet small enough to have adequate airtime for each participant.

In the IGD model, a single dimension of difference is chosen on which to focus, rather than trying to focus on gender, race, religion, and so on, simultaneously. However, attention is paid to the impact of intersecting identities.

Because Coastal City's initial focus was on racial conflict, this program involved training people of color and white people. ${ }^{2}$ Later, trained co-facilitator pairs, with one member from the subordinated group and one from the dominant group, would conduct community-based IGDs. Co-facilitation by cross-identity partners is a key principle of IGDs.

\section{The Program in Coastal City}

The four-phase program sought to develop a cadre of community volunteers to co-facilitate IGDs in Coastal City. Phase one, preparation, involved engaging community leaders and others from a variety of racial and ethnic groups and organizations. Individuals were approached and provided with full information on the values and purposes of the overall IGD effort, as well as its flow, dates, and requirements. After written applications and interviews, twenty-two prospective co-facilitators emerged: three Africans, one African American, four Latinas and Latinos, two Asian Americans, one Native American, and eleven whites, for a total of six men and sixteen women. Some people also identified as multiracial.

Phase two engaged these twenty-two as participants in a 3-day IGD. In order to co-facilitate IGDs, participants needed first to experience an IGD 
themselves, to deepen and accelerate their own awareness and work on racism. Due to budget constraints Lensen conducted and facilitated this event alone. A month later, phase three involved another 3-day workshop, this one focused on learning and practicing the basics of conducting an IGD. Chesler and Brown joined Lensen in co-facilitating this event. Phase four consisted of the conduct of IGDs in Coastal City, led by cross-race pairs of trained co-facilitators, coordinated by a unit of city government, and supported by substantial coaching.

\section{Design of Phase Two}

Phase two began (as did phase three and subsequent community-based IGDs) with introductions, the event's purpose, and suggested norms for working with one another, including openness, risk-taking, and relative safety. We indicated that while IGDs can focus on any one of many dimensions of difference, this process and the initial community IGDs would focus on race and ethnicity. We then moved to personal explorations of individuals' social group identities and the intersections among different aspects of these identities (e.g., race, nationality, gender, sexual orientation, socioeconomic class). Participants reflected on how their identities placed them in positions of advantage or disadvantage regarding access to societal resources and opportunities. They then considered how these identities affected their perceptions and behavior in relationships within and between groups, including what was happening at that moment in the group.

An exercise was introduced to help participants acknowledge and share their personal experiences as "victim," "perpetrator," "bystander," or "intervener" in oppressive situations. This activity led to continued deep sharing of painful experiences and a discussion of recent interactions and conflicts in the room that reflected these four situations.

Participants then explored their understandings of, and reactions to, issues of local controversy. The purpose was not simply to surface individual views, but also to consider how social identities influence these views. Each person then joined with one or more others to develop an action plan to advance social justice locally. As one might expect, participants held disparate views about what constitutes social justice, where key opportunities for action lay, and what actions would be taken. The IGD format provided ample space for exploring these differences. The above-described sequence 
for phase two is the same sequence that would later be used in communitybased IGDs. Thus, by the close of phase two the prospective co-facilitators had experienced as participants the process and challenges that they would later experience as co-facilitators.

\section{Design of Phase Three}

Prospective co-facilitators now focused on learning and practicing IGD theories, methods, and activities. We emphasized that this workshop would be a new learning event, not a continuation of the phase-two IGD, although participants would keep attending to focus on their own growth. Participants practiced co-facilitating with one another, receiving extensive feedback from peers and the consultants.

Key concepts and guidelines for co-facilitation were frequently discussed, including:

- Recognizing and using their own identities as models, resources, and points of connection with participants

- Promoting the expression of differing points of view

- Noticing and interrupting expressions of privilege and oppression in the group as they occur, and encouraging participants to do the same

- Using inquiry methods of challenge and support

- Balancing the voice and power of members of different groups

\section{Phase Four: Community Intergroup Dialogues}

The trained co-facilitators worked in cross-race pairs to lead community dialogues, each IGD spanning 6 to 8 weeks and meeting weekly for 3 to 4 hours. Nine IGDs were conducted in the first 18 months of phase four, involving more than a hundred community participants. Important reinforcement and support included: (1) extensive coaching, (2) periodic cofacilitator learning events, (3) a binder of reference material for each co-facilitator, and (4) feedback from others.

Each co-facilitator pair chose a coach from among Lensen, Chesler, and Brown. Coaching occurred mostly by phone, with coaching conversations before the series started, as the pair planned each session, and after each session 
or two. What had happened in each IGD session was reviewed, and plans for the coming session(s) were discussed. Frequent topics included how the cofacilitators worked together, how racism and other isms were manifesting, signs of small and large progress, moving between one's plan and the group's energy, when to lessen tension and when to let it build, connecting what happened in the group to what happens in Coastal City, how to use one's own race and ethnicity to foster learning, what to do in stuck places, and confidentiality. Since some co-facilitators had significant experience in other forms of dialogue and conflict response, but not in IGD, coaches occasionally needed to tug them back to the IGD purpose and format.

Learning events for the full co-facilitator cadre generated new ideas, encouraged sharing and problem-solving about what was occurring in community dialogues, and sustained the energy of co-facilitators who had been trained but had not yet led community IGDs.

After a few IGDs had been conducted, and at the request of co-facilitators, we created a reference binder of IGD principles and activities. Co-facilitators were encouraged to personalize their copy by noting their observations and experiences and adding variations and new activities.

Feedback from colleagues, family, friends, and community IGD participants proved to be a rich additional source of phase-four affirmation and improvement.

\section{Major Design Components}

Several major design components ran through all four phases:

- Experiential learning

- Power balancing

- Working on our own isms, all the time

- Reinforcement and support

Experiential learning involves engaging in an activity or conversation, reflecting on what happened, and generalizing from those reflections. For instance:

They all line up, twenty-two strong, shoulder to shoulder, silent. The cofacilitator reads the first three of thirty-six statements: "If you do not speak English fluently in U.S. style, move one step back." ... "If the rituals and 
holidays in your family and cultural tradition are mostly the same as those sanctioned by your workplace, move one step forward." ... "If you feel concern about being able to pay for the health care of yourself and those for whom you are responsible, move one step back." Clumping and separation begin, distances growing with each statement, haves and have-nots of privilege surfacing. At the end, three people are out in front of otherswhite man, white woman, black man. They turn and see the others behind them. Silently, people look around the room, seeing others differently than twenty minutes earlier: layers of lives uncovered, assumptions reinforced, challenged, and reconstructed.

Power balancing is essential because hierarchies of privilege and power affect every interaction, including those in IGDs. By challenging prevailing patterns of participation, power balancing seeks to adjust the imbalance that different groups bring with them to the dialogue. Power balancing does not mean being neutral or impartial, but encouraging more socially just interactions. Toward this end, co-facilitator choices include selecting and sequencing activities to highlight power differences, sharing of airtime, and when and how to intervene in participant conversations. Traditional facilitation's presumption of neutrality often allows socially unjust interactions and expressions of power to continue. By contrast, power balancing supports and sustains all voices, especially those usually marginalized in the larger society. Power balancing also underlies IGD's insistence on cross-identity pairs of co-facilitators.

A pair of male co-facilitators (one Asian American, one white) is frustrated that white men repeatedly challenge their decisions. They also notice that participants of color rarely speak. They identify whose voices and what topics seem to dominate the group's time and energy. The co-facilitators then strategize about actions to be taken by one or both of them, to encourage people from varied identities to engage more equitably in the work of the group.

Working on our own isms, all the time, counters the conscious and unconscious prejudices held by those of us born into a racialized, gendered, and classed society. Through these activities and reflective explorations we can reduce the impact of our biases.

A Latino co-facilitator reflects on his interactions with his white woman co-facilitator and the group. He realizes that participants frequently 
praise him for things she has said or done. He braves his embarrassment and points out to participants his collusion with this gendered pattern.

As noted earlier, four key factors comprised reinforcement and support for co-facilitators. We cannot overstate how crucial ongoing support is to the success of social justice efforts. The societal tide favors those with advantage retaining that advantage, and change inherently entails swimming against that tide.

\section{Assessing the Program}

The program attempted to develop a cadre of volunteers to co-facilitate IGDs in Coastal City. Early measures indicate it was successful. Evaluations at the end of the second and third phases demonstrated high satisfaction with the co-facilitator training experiences. Eight months after phase four began, co-facilitators reflected about their experience in e-mails and letters that were again overwhelmingly positive. Some co-facilitators reported insights they had about the local community, racial realities, and the difficulty experienced in discussing race and racism:

I learned how difficult it is for all people to talk about racism, but that it is more difficult for white people to name racism and go into a deep conversation about it.

A dialogue is a unique opportunity for people to delve in and have genuine, real, productive conversations about important topics like racism. It was a very satisfying experience for me and really worth my time.

Other co-facilitators described more generally what they learned about communicating and creating sturdy relationships with people like themselves and with people unlike themselves:

Personally I've learned so much from the dialogue process just being able to hear the perspectives of other people. Some things I've never heard before, and I carry those with me.

I learned that things we consider simple continue to perpetuate racism, such as some cultural differences that initially we think don't have anything to do with racism. We are very attached to some of our cultural practices, even if they are harmful to other people. 
Two years after phase three, co-facilitators again reflected on the program. Their letters described how the workshops and conducting IGDs had deeply impacted their perceptions, behaviors, and lives:

The difference between dialogue and discussion has been very apparent in my personal life, in how I try to understand where another party may view an issue, difference, point of contention, concept, or anything else.... In my professional life these experiences have enabled me to listen and understand more clearly rather than rushing to solve the problem.

Co-facilitators who led community IGDs discussed what they learned in this leadership role in the following terms:

[Co-facilitating an IGD] added a sense of confidence about what my role can be as a white person engaged in anti-racism work. I took leadership and had to trust my judgment and take risks. Co-facilitating built my confidence that I can step up on these issues.

The learning for us co-facilitators was to really listen to what was going on in the room, unpack what was going on. You have to pay attention not only to what you've planned for the session but what's going on at that moment and be able to respond to it.

And some co-facilitators cited the added value of being coached while conducting the community dialogues:

Our coach helped me grow in what I was doing. There is value in hearing a perspective from someone outside the room. The debrief part of it was really valuable in thinking through what happened and where we're headed.

As happens in many limited-resource situations, the sponsoring civic agency was able to conduct only minimal assessments with community IGD participants. Anecdotal information indicates good outcomes: participants were positive about their IGD experience, developed new ways of viewing themselves and racial issues, and made new allies engaging in their chosen change efforts. The conduct of nine community IGDs indicates the efficacy of the training events and the competence and spirit of the trained co-facilitators. Over time, the number of IGDs being conducted has 
decreased. However, as of this writing, Coastal City remains interested in training more dialogue facilitators.

\section{Lessons Learned}

Some lessons involve the entire effort and others particularly co-facilitator training:

- Stay with the IGD model and reinforce its distinctiveness from other forms of conflict response.

- Attend to consultant and co-facilitator demographics: they always affect how we think and behave, how others react to us, and what people learn.

- Address consultant team dynamics, conflicts, and isms.

- Keep in mind that each of us, including those who consider ourselves enlightened, holds unconscious prejudices and racism, our own picture of social justice and our own views of how best to attain it. It is not easy to explore these issues openly with others, nor to ally for joint action.

- Experiencing an IGD before learning to conduct one is essential.

- When starting a change effort, team up with those who are already excited and positive about it. Then strategize how to spread your initial work to those less enthusiastic or more resistant. Stay alert for serendipitous opportunities.

Some lessons apply primarily to the effort's administration, and to the larger community context:

- The competence, wisdom, and commitment of the primary client sponsor are crucial to success.

- Develop deep, sustained political support from diverse constituencies, civic officials, and community leaders.

- Recruit participants for community IGDs with care. Advance planning, creative thought, much work, and a capable infrastructure are required. 
- Use skilled coaches who are experienced in IGD. This increases cofacilitators' learning and the quality of their work.

- Gather data on co-facilitator trainees' and community participants' experiences, views, alliances formed, and actions taken. Use these data to measure progress and improve the effort.

- Expect the unexpected to occur. When responding to the unexpected, strive to manifest the IGD principles and spirit you hope to create in the community.

\section{Implications for Research on Conflict Responses}

We have indicated the need for systematic data on the impact of the training program and the IGDs on co-facilitators and community dialogue participants. This material would enable outcome comparisons among IGDs in different configurations and settings. It would also allow for comparisons between IGDs and other forms of conflict response, in terms of their ability to prepare peace and justice facilitators, and in the long-term impacts and value of such programs.

In closing we offer these words from a Coastal City co-facilitator:

It's a bigger process than any one moment. If you keep your head up and keep moving forward, you'll get there. You'll find it. You're not going to get lost. Trust the process; it'll come through in the end. If you really focus on what we're doing here and trust the process, then it will get you there.

\section{Notes}

1. The form of intergroup dialogue described here is an amalgam of several forms of intergroup dialogue, but has its closest relationship to the IGD model pioneered by the Program on InterGroup Relations, at the University of Michigan, Ann Arbor.

2. We use the term white to refer to those who identify as Caucasian, and the term people of color collectively for those who identify as other than Caucasian (e.g., Latino/a, African American, Asian American, Native American). We recognize that these terms, like most topics related to race, are themselves subjects of debate. 
Kristin Chung-mei Lensen is an organizational development consultant, specializing in intercultural relations and diversity. Ms. Lensen earned her B.A. in Management from Maryhurst University and her M.A. in Intercultural Relations from Antioch University.

Mark Chesler is Emeritus Professor of Sociology and Project Director in the Program on InterGroup Relations at the University of Michigan. He is an active teacher, researcher, and consultant on issues of intergroup dialogue, multicultural teaching pedagogies, and multicultural organizational development.

Nancy Brown serves as an independent resource to organizations, focusing on organizational design, leadership, and the strategic use of diversity. She received her B.A. in Economics from the University of Michigan and currently lives in Ohio and Maine. 